\title{
Mach's Principle of Inertia Is Supported by Recent Astronomical Evidence
}

\author{
Morley B. Bell \\ National Research Council of Canada, Ottawa, Canada \\ Email: morley.bell@nrc-cnrc.gc.ca
}

Received 25 May 2015; accepted 8 September 2015; published 11 September 2015

Copyright (C) 2015 by author and Scientific Research Publishing Inc.

This work is licensed under the Creative Commons Attribution International License (CC BY). http://creativecommons.org/licenses/by/4.0/

(c) (;) Open Access

\begin{abstract}
Inertial mass is detected on Earth only when matter is accelerated or decelerated. Recently evidence has been reported for a low-level velocity oscillation with a period of $39 \pm 1 \mathrm{Mpc}(127 \pm 3$ Myr) superimposed on the Hubble flow. Like the Hubble flow, this oscillation is assumed to be an expansion and contraction of space itself. If space is oscillating as it expands and the Hubble flow contains a superimposed velocity ripple, matter on Earth will experience alternating accelerations and decelerations relative to the rest of the matter in the Universe. The acceleration curve can be obtained from the velocity oscillation curve simply by taking the magnitude of the derivative of the velocity curve and the acceleration curve is found here to have a period of $63.5 \pm 1.5 \mathrm{Myr}$. Evidence has also been claimed recently for a ubiquitous $\sim 62 \pm 3$ Myr periodic fluctuation superimposed on general trends in the fossil biodiversity on Earth. The periods of the acceleration curve oscillation and fossil biodiversity fluctuations are thus identical within the errors. A second, weaker fluctuation is also detected in both the Hubble flow and fossil biodiversity trends. They too have identical periods of $\sim \mathbf{1 4 0}$ Myr. From this excellent agreement, it is argued here that it is the oscillation in the Hubble flow, through an inertia-like phenomenon involving all the matter in the universe that has produced the fluctuations in the fossil biodiversity on Earth. This may represent the first instance where observational evidence supporting Mach's Principle of Inertia has been found.
\end{abstract}

\section{Keywords}

Galaxies, Active-Galaxies, Distances and Redshifts

\section{Introduction}

Ernst Mach argued that inertia could only be explained if all the masses in the universe were somehow connected, and the means by which such action-at-a-distance can occur has had a long history in physics [1] [2]. 
Phipps [3] claimed that Philipp Frank, a Hitler-refugee philosopher of science and physicist who succeeded Einstein in his academic appointment at Prague, attributed to Mach himself the following wording to describe his principle: "When the subway train jerks, it's the fixed stars that throw you down". Phipps further points out that if the fixed stars are doing what Mach claims, they are doing it right now via action-at-a-distance. They are not waiting millennia for signals of any kind to propagate. However, from studying electromagnetic and gravitational field propagation, we conclude that neither of these proceeds instantaneously. Although the Weak Equivalence Principle finds gravitational mass similar to inertial mass, neither is well understood. However, perhaps the most important thing to note is that Inertial Mass is tied to accelerated motion while the former is not. A possible explanation for instantaneous action-at-a-distance that relates to advanced and retarded potentials has been discussed by [4].

To date, there have been no observations found that would suggest that there might be a direct link between two physical phenomena through Mach's Principle of inertia. This may not be surprising since, in Mach's Principle, at least one of the two variables must be all of the rest of the matter in the universe. Recently, evidence was reported for a low-level oscillation with a period of $39 \pm 1$ Mpc superimposed on top of the Hubble flow [5] when intrinsic redshift components were identified and removed from the redshifts of the SNeIa sources studied by [6]. This result is the culmination of many years of work on intrinsic redshifts in quasars and galaxies by us [7]-[13], and by Tifft [14] [15], whose work on galaxies finds similar intrinsic components. When the intrinsic components have been identified, they can be removed. If the source distances are accurate, when the Vcmb velocities of the sources are plotted versus distance, any superimposed oscillation then becomes visible if it is larger than the source peculiar velocities. This can be seen here in Figure 1 and in Fig. 3 and Fig. 4 of [5] and [16] where the oscillation can be seen to be visible over 6 consecutive cycles.

In the Big Bang model, the cosmological component of the redshift of a source is due to the expansion of space and not to the motion of the object through space. Because of this, the cosmological component of the redshift increases by Ho km/s $\mathrm{s}^{-1} / \mathrm{Mpc}^{-1}$, where Ho is the Hubble constant. This produces the Hubble slope. If the

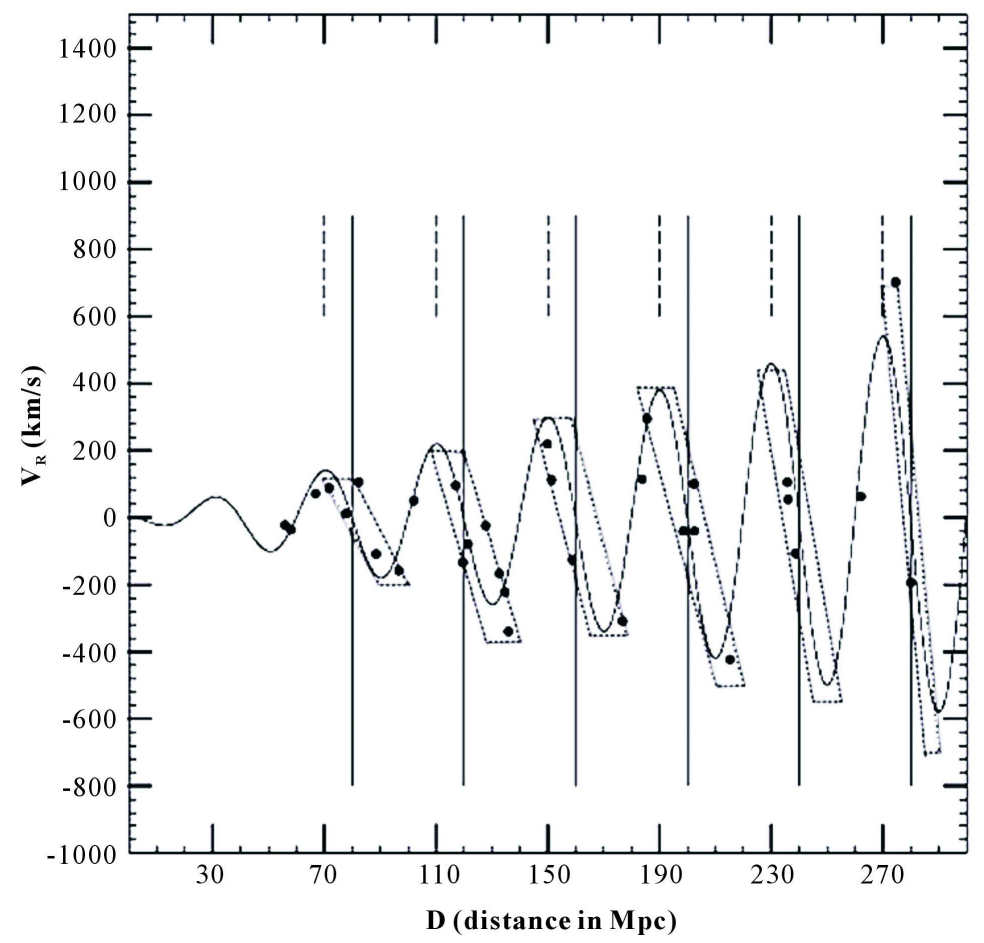

Figure 1. Plot of residual velocity, $\mathrm{V}_{\mathrm{r}}$, versus distance for SNeIa galaxies after removal of intrinsic redshifts and Hubble slope of $57.9 \mathrm{~km} / \mathrm{s}^{-1} / \mathrm{Mpc}^{-1}$ [5] [16]. Regions of high source density are indicated by the dotted rectangles and are centered near 80,120, 160, 200, 240 and $260 \mathrm{Mpc}$, as indicated by the solid vertical lines, and are in phase quadrature with the velocity peaks indicated by the dashed vertical lines. 
velocity oscillation that we have observed is truly an oscillation in the Hubble flow, as proposed here, for a constant amplitude ripple, its observed amplitude also must increase with distance. In Figure 1, which has been reproduced from Fig. 4 of [16], it is clear that the amplitude of the oscillation does increase with distance. As can be seen from Figure 1, the Hubble constant of the ripple is $\mathrm{Hr}=540 / 270=2 \mathrm{~km} / \mathrm{s}^{-1} / \mathrm{Mpc}^{-1}$. Although this increase is expected to continue to much larger distances, it may not be possible to identify the intrinsic components at large distances because of increasing uncertainties in the cosmological parameters. If the intrinsic components cannot be identified and removed, the ripple cannot be detected. Now, for the first time, the oscillations in the Hubble flow will appear to be something that represents all the matter in the universe, as is required to check Mach's Principle. However, to check Mach's Principle, we also require some matter on Earth that may be affected by these oscillations.

Recently a ubiquitous $\sim 62$ Myr periodic fluctuation superimposed on general trends in fossil biodiversity has been reported [17]-[20]. Since the age of the Earth is generally accepted to be $4.5 \mathrm{Gyr}$, detecting fluctuations in the fossil records with a $62 \mathrm{Myr}$ period should not be hindered by the lack of an adequate baseline. Possible associations between this $62 \mathrm{Myr}$ periodicity and the variation of the Sun around the galaxy have been claimed, invoking the modulation of cosmic rays, gamma rays, and comet impact frequency. A major problem with these models is obtaining accurate observational data from them that can be used to compare with the fossil biodiversity fluctuations. An excellent review of what has been done in this area is given by [21], who remain unconvinced that the fossil biodiversity fluctuations are likely to be related to these or other similar Galaxy-related explanations.

In case it is possible that all the matter in the Universe is somehow interconnected, as has been suggested in the inertia phenomenon, one can speculate that the fluctuations in the velocity of the Hubble flow with time might then influence life on Earth in real time, and it is therefore of interest to examine these results more closely. This is especially true in the case of the velocity oscillation where matter on Earth will be alternatively accelerated and decelerated relative to all the rest of the matter in the Universe during each velocity cycle. We now examine if the velocity oscillation we have observed to be superimposed on the Hubble flow might be the true source of the $\sim 62 \mathrm{Myr}$ periodic fluctuation superimposed on general trends in fossil biodiversity.

\section{Analysis}

First we note that the period of the velocity oscillation found to be superimposed on the Hubble flow was 127 Myr, while the fluctuations in the trends in fossil biodiversity on Earth have a period of $62 \mathrm{Myr}$, which differs by approximately a factor of two. However, since inertia is related to acceleration and deceleration, or the rate of change of velocity, of matter, it is more likely, if inertia is involved, that it is the rate of change of velocity of the Hubble flow oscillation (its derivative), that has affected the survival rate of Earth-based genera. Furthermore, it is unlikely that the direction of the change (acceleration or deceleration) would affect things differently and it is therefore more likely that the effects would be related to the magnitude of this parameter.

One cycle of the fluctuations seen in the velocity of the Hubble flow with a period of $127 \mathrm{Myr}$ (39 Mpc) is represented in Figure 2 by the solid curve. The dashed curve shows the magnitude of the derivative of this curve, which represents the magnitude of the rate of change of the velocity. The curve in Figure 3 is the Fourier Transform of the dashed curve in Figure 2 and shows clearly that the dominant frequency present in the dashed curve is $63.5 \mathrm{Myr}$ ( 0.0157 cycles per Myr). This is essentially identical to the ubiquitous $62 \pm 3 \mathrm{Myr}$ periodic fluctuation found to be superimposed on the general trends in fossil biodiversity and, in turn, confirms, as speculated above, that the two are likely to be related. Thus, for the first time, the Hubble oscillation model provides us with observational data involving all the matter in the Universe and a baseline that can be compared directly with that covered in the fossil biodiversity case. When the magnitude of the derivative of the Hubble oscillation is used, which is necessary if inertia is involved, the periods found for the oscillations in the two cases can be seen to be identical within the uncertainties.

\section{Weaker Fluctuations}

Rohde and Muller [17] (see their Fig. 1e) reported that a second fluctuation with a period near $140 \pm 15$ Myrs might also be present in the fossil biodiversity trends. It was also reported previously by us [5] that a weaker fluctuation in the Hubble flow might also be present with a period near 87 Mpc, or 283 Myrs. Again, if it is the magnitude of the derivative of this fluctuation that is affecting the trends in fossil biodiversity we would expect 


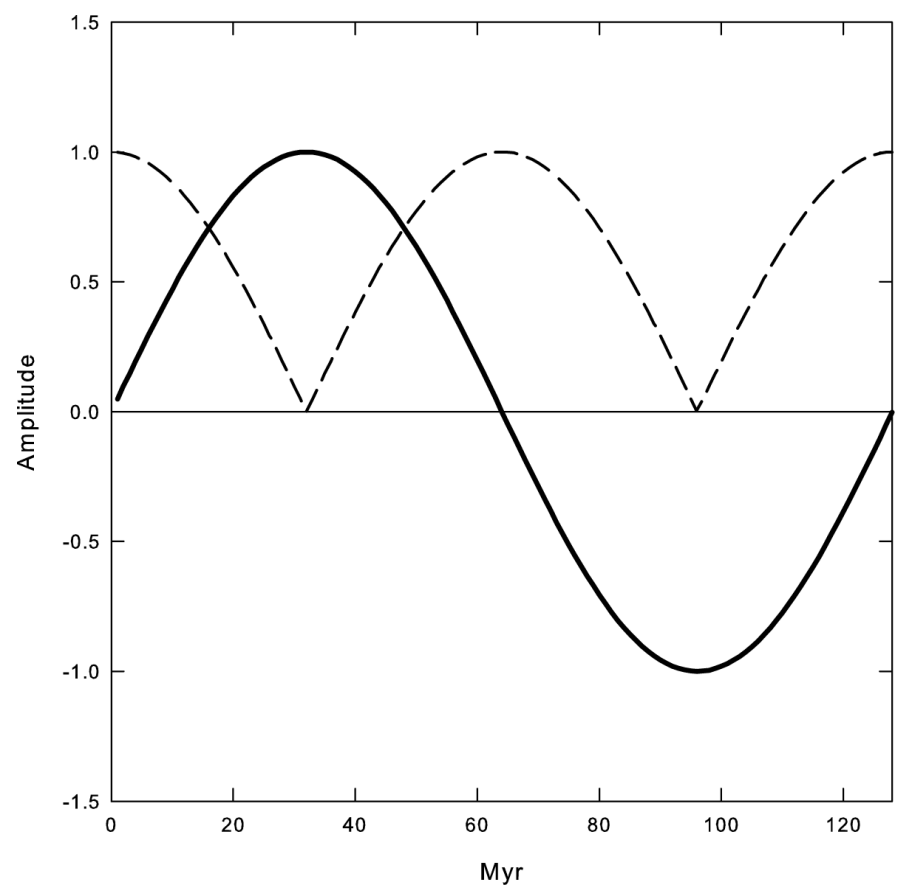

Figure 2. One cycle of the fluctuations seen in the density of the Universe and in the Hubble flow with a period of $127 \mathrm{Myr}$ (39 Mpc) is represented here by the solid curve. The dashed curve shows the magnitude of the derivative of this curve, which then represents the magnitude of the rate of change of the velocity of all the mass in the Universe relative to the matter on Earth.

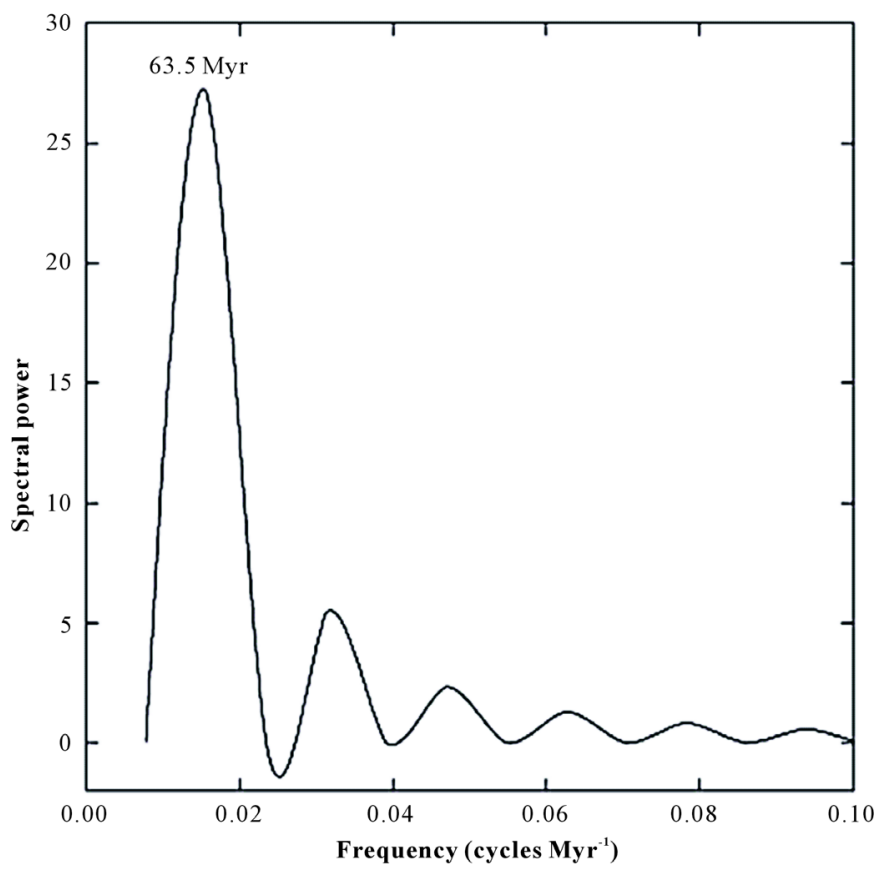

Figure 3. Fourier Transform of the dashed curve in Figure 2 showing clearly that the strongest frequency present in the dashed curve is 0.0157 cycles per Myr, which corresponds to a period of $63.5 \mathrm{Myr}$. This is essentially identical to the ubiquitous $62 \pm 3$ Myr periodic fluctuation found to be superimposed on the general trends in fossil biodiversity. 
it to appear there with a period of one-half this, or 141 Myr. These results are shown in Figure 4 where the curve has been obtained by subtracting best-fit sinusoids from the SNeIa data, recalculating the RMS value, as described previously [5], and converting the fluctuation period from Mpc to Myr using the relation Py (Myr) = [3.26/2] Pp (Mpc). Here the factor 3.26 converts from Mpc to Myr and the factor 2 takes into account the fact that it is the magnitude of the derivative of the fluctuation that is important. In Figure 4 there is excellent agreement between the two periods found in the biodiversity trends (62 and 140 Myrs shown by the vertical dashed lines) and the periods (63.5 and 141 Myrs) found in the Hubble flow assuming that it is the magnitude of the derivative of the oscillation that is the driving force. In both cases (biodiversity and Hubble flow) the short period fluctuations are stronger than the long period ones. This excellent agreement in both period and relative amplitude suggests strongly that it is the oscillation in the Hubble flow that is the source of the fluctuations detected in the fossil biodiversity. The excellent agreement, in both period and relative amplitude, makes it very unlikely that this has occurred by chance.

\section{Instantaneous Communication}

Previously, we [5] [16] used the source distance (look-back time) to show from changes in the density and velocity fields that the Hubble flow was oscillating as it was expanding. This assumed only that the radiation used to detect the sources was propagating at the speed of light. From the results obtained here, if the periodicity seen in the fossil biodiversity is related to changes in the density or velocity fields in the Universe through some kind of inertia-like phenomenon, the effects of these changes would have to be communicated instantaneously. If a communications travel time were involved, such as the speed of light, the time-related effects from matter located at different distances would introduce a smearing effect that would prevent any significant periodic effect on matter located on Earth from being detected. This observation then has the same instantaneous communication requirement as Mach's Principle with the changes in the density and velocity being instantaneously felt by all life types (genera) on Earth.

If this action-at-a-distance is related to the quantum entanglement phenomenon, which requires that all the matter in the Universe at one time had to be connected, we might argue that this picture then agrees well with the Big Bang model. For further discussion see [22].

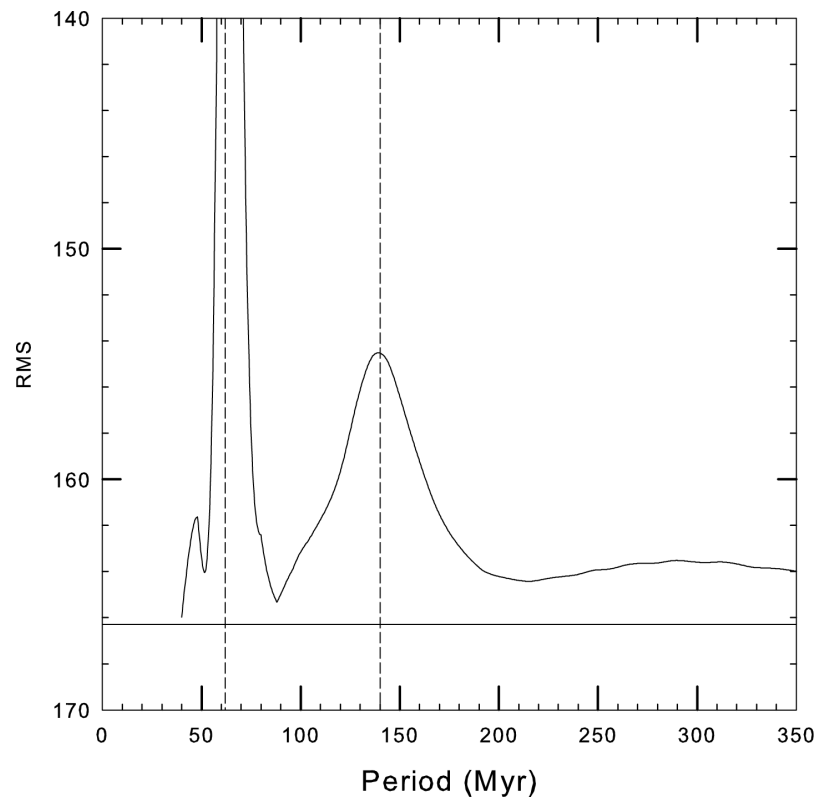

Figure 4. The solid curve represents the ripple peaks found previously for the SNeIa galaxies expressed in millions of years, assuming it is the magnitude of the derivative of the Hubble flow that is the driving force. See text for an explanation of how this curve was obtained. Vertical dashed lines show the periods of 62 and 140 Myr that have already been reported in fossil biodiversity trends. 


\section{Conclusion}

It is shown here that the two dominant periods present in the fluctuations reported in the trends in fossil biodiversity of Earth-based genera (62 \pm 3 and $140 \pm 15 \mathrm{Myr}$ ) are essentially identical to the periods of the derivatives of the two strongest oscillations seen to be superimposed on the velocity of the Hubble flow (63.5 and $141 \mathrm{Myr}$ ). We conclude that it is the oscillation in the Hubble flow which has produced the fossil biodiversity fluctuations. For these effects to be somehow related requires that there be an instantaneous, or action-at-a-distance, relation between the matter on Earth and all the rest of the matter in the universe, as is the case for Mach's Principle. Finally, it is concluded that the results obtained here may be the first empirical evidence supporting Mach's Principle of inertia.

\section{Acknowledgements}

I thank Simon Comeau for comments on the text and assistance with the figures.

\section{References}

[1] Sciama, D.W. (1953) On the Origin of Inertia. Monthly Notices of the Royal Astronomical Society, 113, 34-42. http://dx.doi.org/10.1093/mnras/113.1.34

[2] Hoyle, F. and Narlikar, J.V. (1995) Cosmology and Action-at-a-Distance Electrodynamics. Reviews of Modern Physics, 67, 113-155. http://dx.doi.org/10.1103/RevModPhys.67.113

[3] Phipps, T.E. (1999) Meditations on Action-at-a-Distance. In: Chubykalo, A.E., Pope, V. and Smirnov-Rueda, R., Eds., Instantaneous Action-at-a-Distance in Modern Physics: Pro and Contra, Nova Science, Commack, 137-156.

[4] Narlikar, J.V. (1999) Actions at a Distance in Electrodynamics and Inertia. In: Chubykalo, A.E., Pope, V. and Smirnov-Rueda, R., Eds., Instantaneous Action-at-a-Distance in Modern Physics: Pro and Contra, Nova Science, Commack, 19-34.

[5] Bell, M.B. (2013) Interesting Evidence for a Low-Level Oscillation Superimposed on the Local Hubble Flow. Astrophysics and Space Science, 344, 471-477. http://dx.doi.org/10.1007/s10509-012-1344-7

[6] Freedman, W.L., Madore, B.F., Gibson, B.K., Ferrarese, L., Kelson, D.D., Sakai, S., Mould, J.R., Kennicutt, R.C., Ford, H.C., Graham, J.A., Huchra, J.P., Hughes, S.M.G., Illingworth, J.D., Macri, L.M. and Stetson, P.B. (2001) Final Results from the Hubble Space Telescope Key Project to Measure the Hubble Constant. Astrophysical Journal, 553, 47-72. http://dx.doi.org/10.1086/320638

[7] Bell, M.B. (2002) Evidence for Large Intrinsic Redshifts. Astrophysical Journal, 566, 705-711. http://dx.doi.org/10.1086/338272

[8] Bell, M.B. (2002) Quasar Distances and Lifetimes in a Local Model. Astrophysical Journal, 567, 801-810. http://dx.doi.org/10.1086/338754

[9] Bell, M.B. (2002) Evidence that an Intrinsic Component that is a Harmonic of $z=0.062$ May be Present in Every Quasar Redshift. arXiv:0208320.

[10] Bell, M.B. (2002) Discrete Intrinsic Redshifts from Quasars to Normal Galaxies. arXiv:0211091.

[11] Bell, M.B. and Comeau, S.P. (2003) Intrinsic Redshifts and the Hubble Constant. http://arxiv.org/abs/astro-ph/0305060

[12] Bell, M.B., Comeau, S.P. and Russell, D.G. (2004) Discrete Components in the Radial Velocities of ScI Galaxies. http://arxiv.org/abs/astro-ph/0407591

[13] Bell, M.B. (2007) Further Evidence That the Redshifts of AGN Galaxies May Contain Intrinsic Components. Astrophysical Journal Letters, 667, L129-L132. http://dx.doi.org/10.1086/522337

[14] Tifft, W.G. (1996) Global Redshift Periodicities and Periodicity Structure. Astrophysical Journal, 468, 491-518. http://dx.doi.org/10.1086/177710

[15] Tifft, W.G. (1997) Global Redshift Periodicities and Variability. Astrophysical Journal, 485, 465-483. http://dx.doi.org/10.1086/304443

[16] Bell, M.B. and Comeau, S.P. (2014) More Evidence for an Oscillation Superimposed on the Hubble Flow. Astrophysics and Space Science, 349, 337-442. http://dx.doi.org/10.1007/s10509-013-1601-4

[17] Rohde, R.A. and Muller, R.A. (2005) Cycles in Fossil Diversity. Nature, 434, 208-210.

[18] Melott, A.L. and Bambach, R.K. (2010) A Ubiquitous 62 Myr Periodic Fluctuation Superimposed on General Trends in Fossil Biodiversity: II, Evolutionary Dynamics Associated with Periodic Fluctuation in Marine Diversity. http://arxiv.org/abs/1011.4496 
[19] Melott, A.L. and Bambach, R.K. (2011) A Ubiquitous 62 Myr Periodic Fluctuation Superimposed on General Trends in Fossil Biodiversity: Documentation. Paleobiology, 37, 92-112. http://dx.doi.org/10.1666/09054.1

[20] Melott, A.L. and Bambach, R.K. (2011) A Ubiquitous 62 Myr Periodic Fluctuation Superimposed on General Trends in Fossil Biodiversity: II, Evolutionary Dynamics Associated with Periodic Fluctuation in Marine Diversity. Paleobiology, 37, 383-408. http://dx.doi.org/10.1666/09055.1

[21] Feng, F. and Bailer-Jones, C.A.L. (2013) Assessing the Influence of the Solar Orbit on Terrestrial Biodiversity. Astrophysical Journal, 768, 152-173. http://dx.doi.org/10.1088/0004-637X/768/2/152

[22] Buniy, R.V. and Hsu, S.D.H. (2012) Everything Is Entangled. Physics Letters B, 718, 233-236. http://dx.doi.org/10.1016/j.physletb.2012.09.047 